\title{
Helium lines in RR Lyrae spectra (Research Note)
}

\author{
G. W. Preston \\ Carnegie Observatories, 813 Santa Barbara Street, Pasadena, CA 91101, USA \\ e-mail: gwp@ociw.edu
}

Received 24 July 2009 / Accepted 23 September 2009

\begin{abstract}
Context. During a comparative study of atmospheric phenomena that occur during the pulsation cycles of 10 RRab stars I unexpectedly found helium lines in emission and/or absorption in all 10 stars during rising light. The progression of events in the time-evolution of the helium spectrum differs in detail from star to star, but 9 of the 10 stars share a number of general characteristics, illustrated approximately by the behavior of RV Oct described in this Note.

Aims. My long-term aim is to provide a comprehensive empirical description of atmospheric phenomena that take place during the pulsation cycles of RRab stars. The short-term goal of this paper is to inform readers that measurements of helium lines place new constraints on shock-wave models for RRab stars.

Methods. Results presented here are based on measurements and analysis of observations made with the echelle spectrograph of the du Pont 2.5-m telescope at Las Campanas Observatory.

Results. A general pattern of behavior emerges albeit with significant star-to-star variation. He I $\lambda 5376$ (D3) appears first as a pure emission feature, unlike the Balmer lines for which the emission profile is always overlain initially by strong redward-displaced absorption. The phase of maximum D3 emission coincides approximately with that of $\mathrm{H} \alpha$, but the duration of conspicuous D3 emission (in excess of the local continuum) is less than $15 \mathrm{mn}$, approximately half the duration of similarly defined $\mathrm{H} \alpha$ emission. The emission phase is followed by the appearance, first, of redward-displaced absorption, and shortly thereafter by a second violet-displaced absorption component that strengthens with advancing phase. Both absorption components gradually weaken and disappear after radial-velocity minimum, which lags luminosity maximum by no more than $0.01 \mathrm{P}$ as discussed in Sect. 3. However, bona fide He I $\lambda 5876$ emission flux in excess of the local continuum flux reappears near maximum light for some stars in the sample and persists for about $45 \mathrm{mn}$. The radial velocities of the initial and final He I $\lambda 5876$ emission features differ little from the time-average value of the metallic-line radial velocities for RV Octantis. However, the absorption features appear to distort the location of the emission centroid, when they are strong.
\end{abstract}

Key words. stars: variables: RR Lyr - shock waves

\section{1. introduction}

In this Research Note I report the appearance of helium lines in emission and/or absorption in the spectra of 10 RRab stars during numerous episodes of rising light that have occurred since April 2006. He I $\lambda 5876$ (hereafter D3) emission has long been known to occur in the cepheid-like variable stars of Population II during rising light: in W Vir, first observed by Sanford in the 1940s, as reported by Wallerstein (1959), and in the RV Tau stars U Mon (Preston 1964) and AC Her (Baird 1982). Recently, Schmidt et al. (2004) summarized their spectral survey of cepheids, reporting the presence of D3 emission in a total of nine Population II cepheids. No helium absorption components have been reported in these stars, but it seems possible that this could be a consequence of inadequate phase coverage and/or inadequate spectral resolution.

The ten RRab stars in which helium lines have been found were chosen for study initially to learn how the pulsation characteristics of the peculiar RRab star TY Gru (Preston et al. 2006) compare to those of a random sample of RRab stars with periods near $0.57 \mathrm{~d}$. Accordingly, a program of observations of RRab stars was begun in 2006 with the echelle spectrograph of the du Pont telescope at Las Campanas Observatory.
TY Gru is a Blazhko star, and perchance 5 of the 10 stars selected for study are also Blazhko variables. The Blazhko phenomenon introduces unavoidable complications in the gathering of data and in the inter-comparisons of stars. On the other hand Blazhko stars offer the opportunity to study changes in the behavior of helium lines in individual stars as velocity amplitudes wax and wane during their Blazhko cycles.

\section{The observational data}

In addition to TY Gruis the 10 stars used for comparison are listed in order of increasing pulsation period in Table 1. The $[\mathrm{Fe} / \mathrm{H}]$ values in the 3rd column were taken from Layden (1994). The $V$ and $B$ magnitudes in the 4th and 5th columns of Table 1 were taken from the SIMBAD $^{1}$ database. The numbers of observations accumulated to date for each star (6th column) reflect the complexity of each star's behavior, position in the sky, lunar interference, and weather.

The observations, all made with the $1.5 \times 4$ arcsec entrance aperture of the echelle spectrograph of the du Pont 2.5-m telescope at Las Campanas Observatory, cover the spectral range

1 http://simbad.harvard.edu/simbad/ 
Table 1. RRab stars in whose spectra He I lines appear during rising light.

\begin{tabular}{lllllll}
\hline \hline Star & $P$ & {$[\mathrm{Fe} / \mathrm{H}]$} & $V$ & $B$ & $N_{\text {obs }}$ & Note \\
\hline & & & mag. & mag. & & \\
\hline UV Oct & 0.54260 & -1.61 & 9.0 & 9.8 & 272 & Blazhko \\
AS Vir & 0.55335 & -1.49 & 11.4 & - & 262 & Blazhko \\
V1645 Sgr & 0.55370 & -1.74 & 10.9 & - & 159 & Blazhko \\
DT Hya & 0.56798 & - & 11.7 & - & 102 & - \\
TY Gru & 0.57007 & -2.08 & 14.2 & 14.6 & 177 & Blazhko \\
RV Oct & 0.57116 & -1.34 & 10.9 & 11.2 & 211 & - \\
CD Vel & 0.57349 & - & 11.3 & 11.6 & 208 & Blazhko \\
WY Ant & 0.57433 & -1.66 & 11.0 & 10.8 & 136 & - \\
BS Aps & 0.58256 & -1.33 & - & 12.0 & 256 & Blazhko \\
Z Mic & 0.58693 & -1.28 & 11.2 & 11.6 & 106 & - \\
XZ Aps & 0.58724 & -1.57 & - & 12.3 & 289 & - \\
\hline
\end{tabular}

$\lambda \lambda 3500-9000 \AA$ with resolution $R \sim 27000$ at the $\mathrm{Mg} \mathrm{I} \mathrm{b}$ lines ( $\lambda 5180 \AA$ ), and are more or less uniformly distributed with respect to pulsation phase. Time resolution of 3 to $10 \mathrm{mn}$ with $S / N \sim 20$ at $\lambda 4300 \AA$ is achieved for the 10 comparison stars in Table 1. Spectra for measurement and analysis are created by use of IRAF reduction packages for bias subtraction, flat field division, one dimensional extraction, and for wavelength calibration provided by frequent observation (at least one per hour at each star position) of a Thorium-Argon lamp.

Particular attention has been paid to the phases of rising light $(0.8<\phi<1.0)$; thus, the phase density of the observations during rising light is generally high enough to detect phenomena that occur on a time scale of $0.01 \mathrm{~d}$ for all of the stars in Table 1 except 14th-magnitude TY Gruis, for which time-resolution can only be preserved at the expense of $S / N$. In spite of its faint apparent magnitude D3 emission in the spectrum of TY Gru is well-marked on two occasions. A detailed inter-comparison of the spectroscopic behavior of these eleven stars is underway even as observations continue.

\section{Variation of the D3 line profile with pulsation phase}

The best example of helium emission/absorption phenomena in my sample is illustrated in Fig. 1 by the montage of spectra of RV Oct, obtained during rising light on JD 2454194.5, which traces the progression of changes in the profiles and wavelength displacements of $\mathrm{D} 3$, the sodium $\mathrm{D}$ lines, and $\mathrm{H} \alpha$ with phase, indicated above each spectrum in the left panel. The first seven spectra were observed at approximately $7 \mathrm{mn}$ intervals. To increase $S / N$ as the He features weakened near velocity minimum I co-added two sets of 4 successive observations to produce the bottom two spectra, for which average phases are 0.998 , and 0.043 , respectively. Phases were calculated by use of the ephemeris

$\mathrm{HJD}(\mathrm{RVminimum})=2453835.895+0.571170 * E$.

The zero point of phase is my estimated heliocentric Julian Date of RV minimum, a good surrogate for light maximum. No photometry accompanies my spectroscopic observations. However, from inspection of the best data for $16 \mathrm{RRab}$ stars provided by Cacciari et al. (1987), Jones et al. (1988), Liu \& Janes (1989), and Skillen et al. (1993), I estimate that RV minimum probably lags visual luminosity maximum by a small amount, no greater on average than $0.01 \mathrm{P}$.

The following sequence of events is observed. Beginning at phase 0.920 emission at D3 appears suddenly during rising light and lasts for about $15 \mathrm{mn}$. The largest observed value of peak emission in excess of local normalized continuum is $\sim 0.25$, which must be a lower limit on the true maximum, because the time scale for duration of the emission is comparable to the integration times of the observations, which range from 4 to $15 \mathrm{mn}$. During the $20 \mathrm{mn}$ or so following phase 0.927 the pure emission profile is replaced, successively, by emission plus red-shifted absorption, and briefly by red-shifted absorption alone. At phase 0.953 a blue-shifted absorption component is present and it strengthens relative to the red-shifted component until phase 0.962 . Thereafter, both absorption components gradually weaken with advancing phase past maximum light. At phase 0.998 near maximum light D3 emission above the local continuum reappears weakly between the absorption components and persists for another $\sim 45 \mathrm{mn}$ as discussed in Sect. 4. Thus, for RV Oct D3 is detectable for 0.15 P, or about $2 \mathrm{~h}$, i.e., the He I spectrum is not an evanescent phenomenon.

Among the Blazhko stars in Table 1 (see Kolenberg 2005 for a review of the Blazhko effect) I have detected D3 emission only during those pulsation cycles for which the metal-line radialvelocity amplitude is greater than about $60 \mathrm{~km} \mathrm{~s}^{-1}$, and there are large star-to-star differences among the remaining five stars. For example, I have only detected weak D3 absorption, not clearly resolved into two components in $\mathrm{Z} \mathrm{Mic}$ and in BS Aps during its low-amplitude Blazhko cycles. Finally, emission is not confined to D3; I have found emission lines for the five transitions listed in Table 2, which contains in successive columns the average values and standard deviations for four quantities derived from spectra of RV Oct: radial velocity, emission equivalent width in units of normalized local continuum, $F W H M$ values returned by the IRAF package splot, and peak intensity also measured with splot in units of normalized local continuum. The last column lists the number of observations $n_{\mathrm{obs}}$ used to form each average.

Radial velocity (hereafter RV) measurements for RV Oct are plotted in Fig. 2. The RVs in the top two panels of Fig. 2 are merely Doppler transformations of wavelengths of local extrema in the complex profiles of D3 and $\mathrm{H} \alpha$, measured with the IRAF package splot. By extrema I mean minima in the absorption troughs or local maxima in the apparent emission features between absorption components. When maxima between the double absorption components lie below the local continuum, there is no way to know whether true emission is present or not, so the reality of these "emission peaks" is problematical. Most or perhaps all of these extrema are produced in complicated combinations of emitting and absorbing layers moving with respect to each other, so the velocities calculated from them do not in general indicate true atmospheric motions, as discussed below.

The horizontal lines in all four panels of Fig. 2 represent the time-average velocity, $141.3 \mathrm{~km} \mathrm{~s}^{-1}$, of the smooth curve drawn through the metallic-line velocities (derived from the IRAF package fxcor) plotted in the lower-right panel of Fig. 2. These were obtained by cross-correlation of flattened, normalized spectra, constructed from 13 echelle orders covering the region $\lambda \lambda 4000-4600 \AA$ with the high $S / N$ template spectrum of CS 22874-009 used to conduct the blue metal-poor star binary survey of Preston \& Sneden (2000). RVs derived from wavelengths of the Na I lines, measured with IRAF package splot, are plotted in the lower-left panel of Fig. 2. They are in excellent agreement with the metallic-line fxcor velocities.

$\mathrm{H} \alpha$ emission begins to strengthen in the top spectrum $(\phi=$ 0.913 ) of Fig. 1. Helium emission appears, barely visible, in the second spectrum $(\phi=0.920)$ in which the stellar Na I D lines are noticeably broader $(F W H M=0.70 \AA)$ than those in 
Table 2. Properties of He I lines measured in du Pont echelle spectra of RV Oct.

\begin{tabular}{lllllllllll}
\hline \hline WL & Terms & $\langle\mathrm{RV}\rangle$ & $\sigma$ & $\langle\mathrm{EW}\rangle$ & $\sigma$ & $\langle F W H M\rangle$ & $\sigma$ & $I_{\max }$ & $\sigma$ & $n$ \\
\hline$(\AA)$ & & $\left(\mathrm{km} \mathrm{s}^{-1}\right)$ & $\left(\mathrm{km} \mathrm{s}^{-1}\right)$ & $(\AA)$ & $(\AA)$ & $\left(\mathrm{km} \mathrm{s}^{-1}\right)$ & $\left(\mathrm{km} \mathrm{s}^{-1}\right)$ & & $(\AA)$ & \\
\hline 4471.48 & $2^{3} \mathrm{P}-4^{3} \mathrm{D}$ & 144.6 & 4.2 & 0.052 & 0.006 & 51.6 & 3.8 & 0.071 & 0.007 & 4 \\
5875.62 & $2^{3} \mathrm{P}-3^{3} \mathrm{D}$ & 135.6 & 0.8 & 0.200 & 0.015 & 43.6 & 0.7 & 0.226 & 0.011 & 5 \\
6678.15 & $2^{1} \mathrm{P}-3^{1} \mathrm{D}$ & 133.7 & 2.7 & 0.096 & 0.011 & 42.1 & 2.1 & 0.098 & 0.011 & 5 \\
7065.19 & $2^{3} \mathrm{P}-3^{3} \mathrm{~S}$ & 129.8 & 2.2 & 0.053 & 0.006 & 42.9 & 9.6 & 0.048 & 0.015 & 5 \\
7281.48 & $2^{1} \mathrm{P}-3^{1} \mathrm{~S}$ & 121.5 & 9.6 & 0.040 & 0.014 & 43.3 & 4.8 & 0.027 & 0.005 & 4 \\
\hline
\end{tabular}
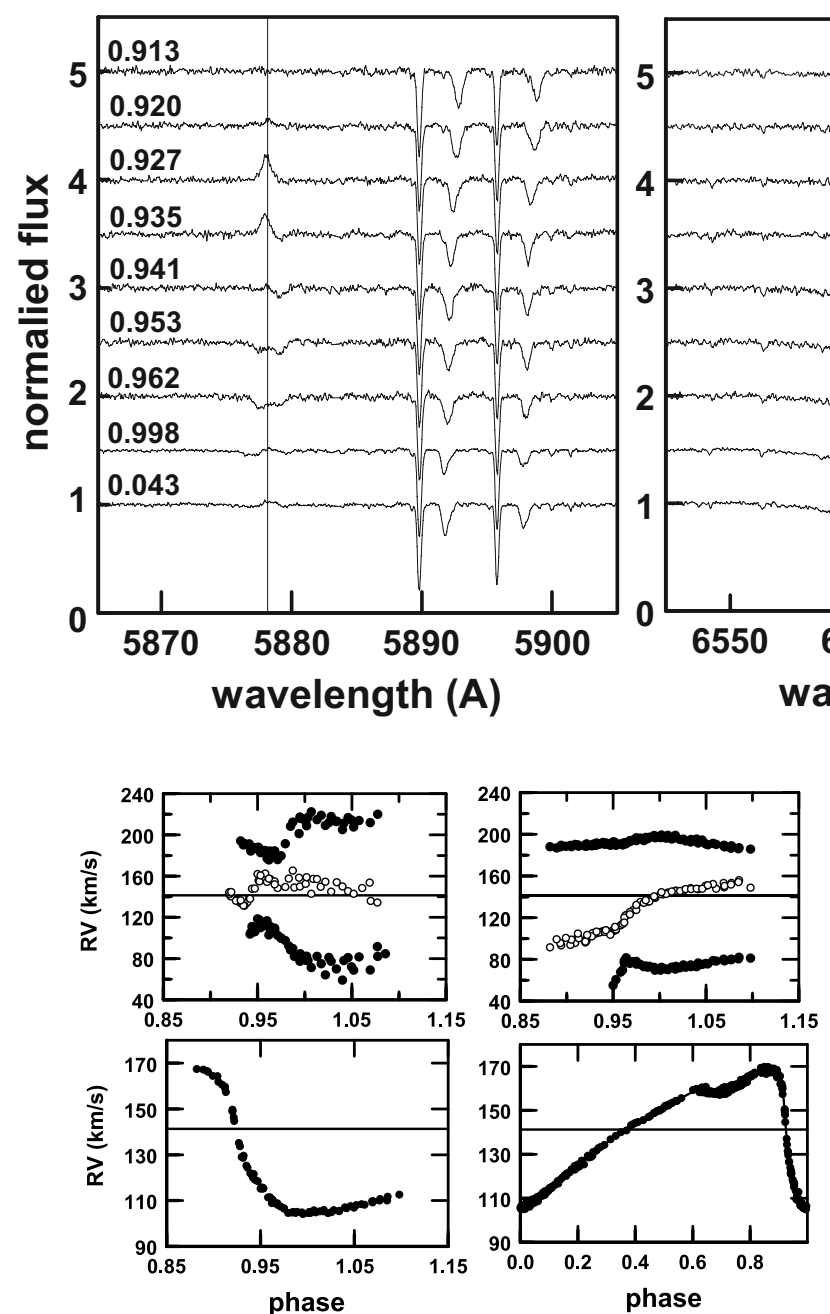

Fig. 2. Radial velocities for RV Oct on JD 2454194.5 calculated from wavelengths of extrema in the absorption/emission profiles as discussed in the text: (upper left) for D3; (upper right) for $\mathrm{H} \alpha$; (lower left) for the $\mathrm{Na}$ I D lines adjacent to D3; (lower right) for metal lines derived from fxcor as described in the text. Filled and open circles in the upper panels denote absorption and emission "velocities", respectively. Horizontal lines at $141.3 \mathrm{~km} \mathrm{~s}^{-1}$ in each panel denote the time-average velocity derived from a smooth curve drawn through the metal line data.

the immediately preceding $(F W H M=0.66 \AA)$ and following $(F W H M=0.64 \AA)$ spectra. D3 emission reaches maximum strength sometime during the 3rd and 4th exposures $(\phi=0.927$ and 0.935), as does the $\mathrm{H} \alpha$ emission. The D3 emission line in the first of these, apparently uncontaminated by red-shifted absorption, yields an RV that is closely equal to the time-average RV derived from the metal-lines, which I use as a rough approximation for the center of mass velocity of the star (Bono et al. 1994). Thus, when it first appears, the D3 emission centroid lies near the center-of-mass velocity of the star, as surmised long ago (Preston et al. 1965, p. 131) for the hydrogen emission in RR Lyrae. So, it appears that at midrising light we observe a shock that is approximately stationary with respect to the center of mass of the star, consistent with the pictures presented by Gillet \& Crowe (1988) for X Ari, and most recently by Chadid et al. (2008) for $S$ Arae: the shock initially appears to move inward, stop, and then advance outward on a timescale of minutes. For whatever reasons I have failed to detect these systematic in and out motions of the emitting gas.

The appearance of red-shifted absorption in the fifth panel $(\phi=0.941)$ "pushes" the measured emission initially to spurious more negative velocities in the top left panel of Fig. 2. This RV trend is quickly reversed when a second rapidly strengthening violet absorption appears in the 6th and 7 th spectra $(\phi=0.953$ and 0.962). Analogous, much more pronounced effects, seen in the $\mathrm{RV}$ behavior of $\mathrm{H} \alpha$ in the top-right panel of Fig. 2, can be interpreted in the same way: strong red-shifted overlying $\mathrm{H} \alpha$ absorption present in all the spectra preceding maximum light greatly distorts the $\mathrm{H} \alpha$ emission profiles and velocities calculated from their extrema.

\section{Post-maximum helium emission}

Perhaps the most surprising aspect of these observations is the reappearance of helium emission, which becomes clearly visible a second time near maximum light $(\phi=0.000)$, then slowly weakens and disappears after about $45 \mathrm{mn}$. To illustrate this emission, barely recognizable in the bottom spectrum of Fig. 1, I co-added eighteen spectra of RV Oct obtained in the phase interval $0.00 \leqslant \phi \leqslant 0.06$ to obtain the spectrum displayed in Fig. 3 . The D3 flux during emission maximum exceeds that of the local 


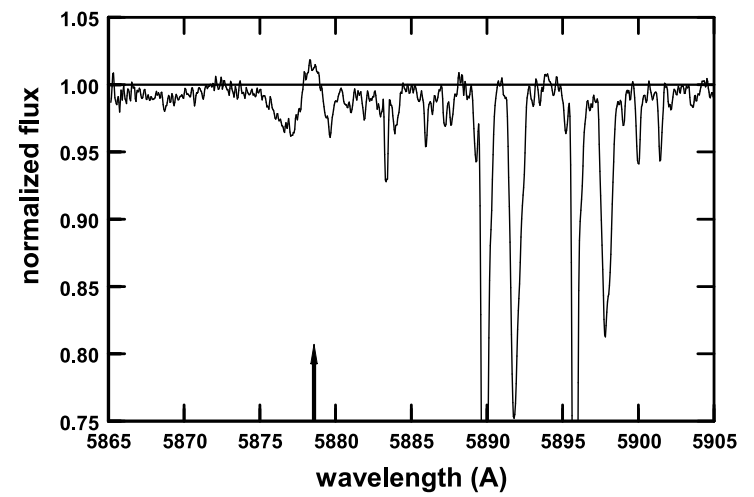

Fig. 3. The profile of D3 in the spectrum of RV Oct created by co-adding eighteen spectra obtained in the phase interval $0.00 \leqslant \phi \leqslant 0.06$. The position of D3 in the red-shifted spectrum of RV Oct is marked by a vertical arrow.

continuum flux by four times the rms pixel-to-pixel flux variation in the nearby continuum. The measured RVs of individual observations used to construct this spectrum all lie red-ward of the time-average metallic-line velocity by $\sim 10 \pm 8 \mathrm{~km} \mathrm{~s}^{-1}$ (see Fig. 2, upper left panel). I do not know how to estimate the distortions of measured velocities that might be produced by flanking violet-displaced absorptions. It is conceivable to me that the entire $10 \mathrm{~km} \mathrm{~s}^{-1}$ offset could be produced by such distortion, in which case stationary helium emission may be present throughout the light-rise, only becoming recognizable as such after overlying absorption weakens sufficiently. Analogous weak hydrogen emission, if present, is masked by overpowering absorption at these phases.

Fokin \& Gillet (1997) present an awesome calculation of shocks and compression waves that occur during an RR Lyr pulsation cycle (see their Fig. 4). Two of their shocks (s1 and s2) are present simultaneously at and immediately after maximum light. I can only speculate that the appearances of helium emission at these phases may be a manifestation of these shocks. In a subsequent paper Fokin et al. (1999) conclude that the maximum in $F W H M$ that occurs just after maximum light is due to a velocity gradient in the line-producing layers rather than to unresolved shock line-doubling. The helium emission/double absorption profile that persists after phase 0.00 suggests that shock activity may be a more important contributor to atomic absorption line contours at these phases than had been realized previously.

\section{Discussion}

The nature of the He I spectrum in RRab stars differs substantially from that of W Vir (Lèbre \& Gillet 1992) with respect to the numbers of lines detected thus far, the phase-duration of emission, particularly the post-maximum light emission at D3, and the complex time-evolution of the associated absorption components. In these respects, the RR Lyrae stars present a rich source of observational material with which to explore atmospheric structure during the shock-wave phases of their pulsation cycles. Although the behavior of the He and $\mathrm{H}$ lines conform in a general way to the shock model proposed by Schwarzschild (1952), the He lines add important detail. Because of the large excitation and ionization potentials of helium relative to those of hydrogen, the appearance and disappearance of helium lines on a time scale of $\sim 2 \mathrm{~h}$ add new constraints on the rapidly evolving temperature structure of the atmosphere during the "hump" near phase 0.93 (Gillet \& Crowe 1988), and raise questions about the persistence of emission long after maximum light. Successful models must account for emission fluxes that arise in or near the shock as well as absorption line strengths produced by hydrogen and helium in the infalling and outflowing layers. The effect of shock waves on $\mathrm{H} \alpha$ profiles in RR Lyrae spectra has been modeled by Fokin (1992), but he made no predictions about helium in emission or absorption. A theoretical treatment of He I lines that occur under the conditions of temperature and density encountered in RRab atmospheres near rising light would be most welcome.

Finally, I call attention to the apparent decrease in RV with wavelength in Cols. 1 and 3 of Table 2 with some diffidence. Paschen bound-free absorption is the dominant source of atmospheric opacity at optical wavelengths in the atmosphere of an RRab star during rising light $\left(T_{\text {eff }} \sim 6500 \mathrm{~K}\right)$; it increases by a factor of about 5 between $4000 \AA$ and $8000 \AA$. Interpreted as a level effect produced by this opacity gradient, the velocity differences in Table 2 suggest that during the phase of strong helium emission the deepest layers of the atmosphere that contribute to $\lambda 4471$ are stationary in the rest frame of the star, while superincumbent layers are moving outward at a rate that increases with distance above the photosphere. Further discussion of this preliminary result best awaits confirmation by observations with higher $S / N$, and superior time resolution that will permit study of the time-evolution of such velocity structure.

The observations reported here were recorded with lengthy (93 s) readout times of a $2048 \times 1500$ pixel raster in order to maximize echelle spectral coverage at a telescope of modest $(2.5 \mathrm{~m})$ aperture. Integration times of my du Pont observations vary with pulsation phase, star brightness, and observing conditions, and they range from $200 \mathrm{~s}$ to $1000 \mathrm{~s}$ for the stars in Table 1. Order-ofmagnitude improvement in time-resolution (to isolate the phase of maximum emission strength) and $S / N$ (to reduce the substantial errors in continuum location, hence emission flux) could be achieved by restricting read-out to small portions of adjacent echelle orders that contain, for example, $\lambda 6678$ and $\lambda 7281$, and by use of telescopes of larger (6 to $10 \mathrm{~m}$ ) aperture.

Acknowledgements. The author wishes to thank referee Dr. Philippe Mathias for his many helpful comments, questions, and suggestions that greatly improved this Research Note.

\section{References}

Baird, S. R. 1982, PASP, 94, 850

Bono, G., Caputo, F., \& Stellingwerf, R. F. 1994, ApJ, 432, L51

Cacciari, C., Clementini, G., Prévot, L., et al. 1987, A\&AS, 69, 135

Chadid, M., Vernin, J., \& Gillet, D. 2008, A\&A, 491, 537

Fokin, A. B. 1992, MNRAS, 256, 26

Fokin, A. B., \& Gillet, D. 1997, A\&A, 325, 1013

Fokin, A. B., Gillet, D., \& Chadid, M. 1999, A\&A, 344, 930

Gillet, D., \& Crowe, R. A. 1988, A\&A, 199, 242

Jones, J. V., Carney, B. W., \& Latham, D. W. 1988, ApJ, 332, 206

Kolenberg, K. 2005, ASP Conf. Ser., 335, 95

Layden, A. 1994, AJ, 108, 1016

Lèbre, A., \& Gillet, D. 1992, A\&A, 255, 221

Liu, T., \& Janes, K. A. 1989, ApJS, 69, 593

Preston, G. W. 1964, ApJ, 140, 173

Preston, G. W., \& Sneden, C. 2000, AJ, 120, 1014

Preston, G. W., Smak, J., \& Paczynski, B. 1965, ApJS, 12, 99

Preston, G. W., Thompson, I. B., Sneden, C., Stachowski, G., \& Shectman, S. A. 2006, AJ, 132, 1714

Schwarzschild, M. 1952, Transactions IAU VIII, ed. P. Th. Oosterhoff, (Cambridge U. Press), 811

Schmidt, E. G., Johnston, D., Lee, K. M., et al. 2004, AJ, 128, 2988

Skillen, I., Fernley, J. A., Stobie, R. S., et al. 1993, MNRAS, 265, 301

Sneden, C., Preston, G. W., \& Cowan, J. J. 2003, ApJ, 592, 504

Wallerstein, G. 1959, ApJ, 130, 560 